\title{
COMBINED EMPIRICAL AND ANALYTICAL VIRTUAL SENSING IN STRUCTURAL DYNAMICS FOR UNCERTAINTY REDUCTION
}

\author{
Jyrki Kullaa \\ Helsinki Metropolia University of Applied Sciences \\ P.O. Box 4021, 00079 Metropolia, Finland \\ e-mail: jyrki.kullaa@metropolia.fi
}

Keywords: Virtual sensing, Structural dynamics, Response estimation, Sensor network, Strain field estimation, Uncertainty reduction.

\begin{abstract}
Structural monitoring and control utilize vibration measurements acquired by a sensor network. For example, fatigue assessment uses stress or strain histories at critical locations of the structure. However, some locations may be impractical or inaccessible for instrumentation. Therefore, accurate estimation of the response at these locations using available measurements would be useful.
\end{abstract}

Analytical virtual sensing techniques use information available from a sensor network together with a finite element model to calculate an estimate of the unmeasured quantities of interest. In linear structural dynamics, the response of the structure can be expressed as a sum of modal contributions. Therefore, the analytical mode shapes from the finite element model can be used as a basis to estimate the response.

Empirical virtual sensing uses only measurement data to build a regression model of the sensor network. If the sensor network is redundant, the signal of a single sensor can be estimated from the remaining sensor network data using e.g. minimum mean square error (MMSE) estimation.

A combined algorithm uses both empirical and analytical virtual sensing to approximate the full-field data from a limited set of sensors. Because of measurement errors, the estimation must be performed from noisy measurements. First, empirical virtual sensing is applied to estimate less noisy sensor signals that will replace the measurement data. The derived error covariance matrix is used as a weighting matrix in subsequent analytical virtual sensing.

Different approaches were compared in a numerical study: ordinary least squares and weighted least squares using either analytical or combined virtual sensing. Combined virtual sensing outperformed analytical virtual sensing in a large sensor network or in the case of high measurement error. 


\section{INTRODUCTION}

Sensors and monitoring systems occur everywhere. Current technology makes it possible to install a wireless sensor network with a high number of low-cost small sensors. An important application is structural monitoring, which utilizes vibration measurements acquired by a sensor network to assess the condition of structures. For example, fatigue assessment uses stress or strain histories at critical locations of the structure. However, some locations may be impractical or inaccessible for instrumentation. Therefore, estimating the response at these locations using available measurements could be useful for fatigue assessment. Sensors can also be used for vibration control.

Virtual sensing techniques estimate unmeasured quantities using the available physical sensors. In vibration monitoring, the response can be assumed to consist of the sum of modal contributions, in which only a few natural modes are active. With this assumption, a finite number of sensors is enough to make the sensor network redundant. This redundancy can be utilized in the estimation.

Virtual sensing can be either analytical (model-based) or empirical (data-driven) [1]. Analytical virtual sensing techniques use information available from a limited set of physical sensors together with the finite element model to calculate an estimate of the quantity of interest. For example, it is possible to estimate the stress or strain field from acceleration measurements. Analytical mode shapes from the finite element model are typically used as a basis to estimate the response at unmeasured locations by an expansion algorithm [2].

Some challenges exist in analytical virtual sensing: The estimation accuracy may suffer from the measurement noise. This is especially true for wireless sensor networks (WSN) with low-cost sensors. Moreover, the measurement noise may be unknown. The excitation or other environmental loads are often difficult to measure for civil engineering structures, and the response is only measured. In addition to the measurement noise, the model accuracy, or specifically the accuracy of the mode shapes, affects the accuracy of the virtual sensors.

Analytical virtual sensing in structural dynamics has been studied e.g. in the following papers. Avitabile [2] applied virtual sensing to correlation of analytical and experimental models using model expansion. Sestieri et al. [3] estimated rotational DOF from a limited set of measured translational DOF. Hjelm et al. [4] estimated stress histories from acceleration measurements. Iliopoulos et al. [5] applied virtual sensing to fatigue assessment of wind turbines. Full-field dynamic stress/strain field was estimated from limited sets of measurement data in [6, 7]. Maes et al. [8] compared different response estimation algorithms.

Empirical virtual sensing can be used to replace a temporarily installed or failed sensor [9]. Empirical virtual sensing has also been used for damage or sensor fault detection [10]. Also, the estimation error can be evaluated. The number of sensors in WSN is often large, which can increase the accuracy of estimation.

A combined virtual sensing algorithm is introduced, which applies both empirical and analytical virtual sensing to obtain an estimate of the quantity of interest. The objective is to reduce uncertainty of virtual sensing techniques due to measurement noise. A redundant sensor network is assumed. The model error is ignored in this paper. Although an exact FE model is not realistic, it is possible to isolate the measurement error as the sole source of error thus facilitating the comparison of different approaches.

The paper is organized as follows. Empirical virtual sensing and analytical virtual sensing are introduced in Section 2 with a proposed combination of either technique for uncertainty reduction. Numerical study is performed in Section 3 with noisy vibration measurements to validate the proposed method. Concluding remarks are given in Section 4. 


\section{VIRTUAL SENSING}

Virtual sensing (VS), or soft sensing, is used to provide an alternative to physical measurement instrument. The quantity of interest is estimated using the available measurements and the system model. Virtual sensing can be classified into empirical and analytical techniques.

\subsection{Empirical virtual sensing}

Empirical virtual sensing is based on available historical measurements. These data are used to estimate the correlation between the measured quantities. Empirical virtual sensing uses regression techniques that can be implemented using different statistical methods, for example linear regression or neural network regression.

Minimum mean square error (MMSE) estimation [10] uses the data covariance matrix to estimate one or more variables using the remaining variables. Here, the variables are simultaneously sampled sensor measurements $\mathbf{x}_{m}(t)$. The time instant $t$ will be omitted in the sequel: for example, $\mathbf{x}_{m}(t)$ will be written shortly as $\mathbf{x}_{m}$.

With enough redundancy, a subset of observation $\mathbf{x}_{m}$ can be estimated using the remaining variables. Each observation is divided into observed variables $\mathbf{v}$ and missing variables $\mathbf{u}$ :

$$
\mathbf{x}_{m}=\left\{\begin{array}{l}
\mathbf{u} \\
\mathbf{v}
\end{array}\right\}
$$

with a partitioned mean vector $\mu$ and data covariance matrix $\Sigma$ :

$$
\boldsymbol{\mu}=\left\{\begin{array}{l}
\boldsymbol{\mu}_{u} \\
\boldsymbol{\mu}_{v}
\end{array}\right\} \quad \boldsymbol{\Sigma}=\left[\begin{array}{ll}
\boldsymbol{\Sigma}_{u u} & \boldsymbol{\Sigma}_{u v} \\
\boldsymbol{\Sigma}_{v u} & \boldsymbol{\Sigma}_{v v}
\end{array}\right]=\left[\begin{array}{ll}
\boldsymbol{\Gamma}_{u u} & \boldsymbol{\Gamma}_{u v} \\
\boldsymbol{\Gamma}_{v u} & \boldsymbol{\Gamma}_{v v}
\end{array}\right]^{-1}
$$

where the precision matrix $\Gamma$ is defined as the inverse of the covariance matrix $\Sigma$ and is also written in the partitioned form. A linear MMSE estimate for $\mathbf{u} \mid \mathbf{v}$ ( $\mathbf{u}$ given $\mathbf{v}$ ) is obtained by minimizing the mean-square error (MSE) and can be computed either using the covariance or precision matrix [10]. If each sensor is estimated in turn, using the precision matrix results in a more efficient algorithm. The expected value of the missing variable is:

$$
\hat{\mathbf{u}}=E(\mathbf{u} \mid \mathbf{v})=\boldsymbol{\mu}_{u}-\boldsymbol{\Gamma}_{u u}^{-1} \boldsymbol{\Gamma}_{u v}\left(\mathbf{v}-\boldsymbol{\mu}_{v}\right)
$$

and the error covariance MSE is

$$
\operatorname{cov}(\mathbf{u} \mid \mathbf{v})=\Gamma_{u u}^{-1}
$$

If all variables are estimated in turn using the remaining variables, the expected values of all variables can be written in matrix form [11]:

$$
\hat{\mathbf{x}}_{m}=\boldsymbol{\mu}+\mathbf{A}\left(\mathbf{x}_{m}-\boldsymbol{\mu}\right)
$$

where the coefficient matrix $\mathbf{A}$ is composed as follows.

For simplicity, assume zero-mean variables $\mathbf{x}_{m}$. Let us consider variable $x_{k}$ to be estimated:

$$
\hat{\mathbf{u}}=\hat{x}_{k}=E(\mathbf{u} \mid \mathbf{v})=-\boldsymbol{\Gamma}_{u u}^{-1} \boldsymbol{\Gamma}_{u v} \mathbf{v}=\mathbf{b}^{T} \mathbf{v}
$$

where $\mathbf{b}^{T}=-\boldsymbol{\Gamma}_{u u}^{-1} \boldsymbol{\Gamma}_{u v}$ is a $1 \times(p-1)$ row vector 


$$
\mathbf{b}^{T}=\left[\begin{array}{llll}
b_{1} & b_{2} & \cdots & b_{p-1}
\end{array}\right]
$$

Equation (6) can be transformed into the following form introducing all variables $\mathbf{x}$ :

$$
\mathbf{b}^{T} \mathbf{v}=\mathbf{c}^{T} \mathbf{x}
$$

where

$$
\mathbf{v}=\left[\begin{array}{lllllll}
x_{1} & x_{2} & \cdots & x_{k-1} & x_{k+1} & \cdots & x_{p}
\end{array}\right]^{T}
$$

Thus,

$$
\mathbf{c}^{T}=\left[\begin{array}{llllllll}
b_{1} & b_{2} & \cdots & b_{k-1} & 0 & b_{k} & \cdots & b_{p-1}
\end{array}\right]
$$

which is a $1 \times p$ row vector and becomes the $k$ th row of matrix $\mathbf{A}$. The estimation is applied to each variable $x_{k}, k=1,2, \ldots, p$. Hence, each diagonal entry of matrix $\mathbf{A}$ is equal to zero.

The error variances (4) could be used to construct a diagonal weighting matrix, but a full weighting matrix is more efficient, because the residuals may be correlated. The residual covariance matrix $\mathbf{W}$ is derived in the following. Assuming a redundant sensor network with noise $\mathbf{e}(t)$, the sensor network model is

$$
\mathbf{x}_{m}=\mathbf{A} \mathbf{x}_{m}+\mathbf{e}
$$

Thus,

$$
\mathbf{e}=(\mathbf{I}-\mathbf{A}) \mathbf{x}_{m}
$$

where $\mathbf{I}$ is a $p \times p$ unit matrix. The error covariance matrix is

$$
\mathbf{W}=E\left(\mathbf{e e}^{T}\right)=(\mathbf{I}-\mathbf{A}) E\left(\mathbf{x}_{m} \mathbf{x}_{m}^{T}\right)(\mathbf{I}-\mathbf{A})^{T}=(\mathbf{I}-\mathbf{A}) \mathbf{\Sigma}(\mathbf{I}-\mathbf{A})^{T}
$$

This covariance matrix can be used as a weighting matrix in subsequent analytical virtual sensing. $E(\mathbf{u} \mid \mathbf{v})$ will be substituted for the noisy measurement.

\subsection{Analytical virtual sensing}

Analytical virtual sensing uses a mathematical model of the system together with available measurements to estimate the quantity of interest. In this study, ordinary least squares and weighted least squares estimation are investigated.

In linear structural dynamics, the structural response can be written as a sum of modal contributions:

$$
\mathbf{x}(t)=\mathbf{\Phi q}(t)=\sum_{i=1}^{N} \phi_{i} q_{i}(t) \approx \sum_{i=1}^{n} \phi_{i} q_{i}(t)
$$

where $\mathbf{x}(t)$ is the displacement response, $N$ is the number of DOF in the finite element model, $n<<N$ is the selected number of active modes, $\Phi$ is the modal matrix consisting of the mode shapes $\phi_{i}$ as columns, and $\mathbf{q}(t)$ are the modal, or generalized coordinates.

The System Equivalent Reduction Expansion Process (SEREP) algorithm [2] is briefly outlined. If $\mathbf{x}(t)$ is divided into measured and unmeasured DOF, also the mode shape vectors are divided correspondingly: 


$$
\mathbf{x}(t)=\left\{\begin{array}{l}
\mathbf{x}_{m}(t) \\
\mathbf{x}_{u}(t)
\end{array}\right\}=\left[\begin{array}{l}
\boldsymbol{\Phi}_{m} \\
\boldsymbol{\Phi}_{u}
\end{array}\right] \mathbf{q}(t)
$$

The upper equation reads

$$
\mathbf{x}_{m}(t)=\boldsymbol{\Phi}_{m} \mathbf{q}(t)
$$

If the number of sensors is greater than the number of active modes $n$, the modal coordinates $\mathbf{q}(t)$ can be solved using ordinary least squares (OLS) solution

$$
\hat{\mathbf{q}}(t)=\left(\boldsymbol{\Phi}_{m}^{T} \boldsymbol{\Phi}_{m}\right)^{-1} \boldsymbol{\Phi}_{m}^{T} \mathbf{x}_{m}(t)
$$

or weighted least squares (WLS) solution [12]:

$$
\hat{\mathbf{q}}(t)=\left(\boldsymbol{\Phi}_{m}^{T} \mathbf{W}^{-1} \boldsymbol{\Phi}_{m}\right)^{-1} \boldsymbol{\Phi}_{m}^{T} \mathbf{W}^{-1} \mathbf{x}_{m}(t)
$$

where the weighting matrix $\mathbf{W}$ is obtained from (13). Once the estimate of $\mathbf{q}(t)$ is solved, any other DOF can be computed from the lower equation in (15):

$$
\hat{\mathbf{x}}_{u}(t)=\boldsymbol{\Phi}_{u} \hat{\mathbf{q}}(t)
$$

The mode shapes typically consist of displacement and rotation DOFs. They can also be augmented with strains or stresses at different locations of the structure, because the displacement pattern determines the strain or stress distribution.

The error covariance of the WLS estimate of $\mathbf{q}(t)$ (18) is [12]:

$$
\mathbf{C}=\left(\boldsymbol{\Phi}_{m}^{T} \mathbf{W}^{-1} \boldsymbol{\Phi}_{m}\right)^{-1}
$$

Because the unmeasured quantities $\mathbf{x}_{u}(t)$ are linear functions of $\mathbf{q}(t)$ according to Equation (19), their error variances are

$$
\operatorname{var}\left[\hat{\mathbf{x}}_{u}(t)\right]=\operatorname{diag}\left(\boldsymbol{\Phi}_{u} \mathbf{C} \boldsymbol{\Phi}_{u}^{T}\right)
$$

\subsection{Combined empirical and analytical virtual sensing}

In the combined virtual sensing, both empirical and analytical VS are used to obtain a more accurate estimate of the quantity of interest. First, empirical virtual sensing is used to estimate a less noisy measurement for each sensor in turn using the remaining (noisy) sensor network. The MMSE estimate will then replace the noisy measurement. Also, the error covariance, or the weighting matrix $\mathbf{W}$ is obtained at this stage.

The subsequent step is analytical virtual sensing, in which the lowest mode shapes from a finite element model form the basis for estimation. The modal coordinates $\mathbf{q}(t)$ are solved using ordinary least squares or weighted least squares. Then the response at any DOF can be computed.

The combined empirical and analytical virtual sensor reading is derived using (19) and applying either OLS (17) or WLS (18), where $\mathbf{x}_{m}$ is replaced with $\hat{\mathbf{x}}_{m}$ (5). Assuming zero means, the WLS solution is: 


$$
\begin{aligned}
\hat{\mathbf{x}}_{u}(t) & =\boldsymbol{\Phi}_{u} \hat{\mathbf{q}}(t) \\
& =\boldsymbol{\Phi}_{u}\left(\boldsymbol{\Phi}_{m}^{T} \mathbf{W}^{-1} \boldsymbol{\Phi}_{m}\right)^{-1} \boldsymbol{\Phi}_{m}^{T} \mathbf{W}^{-1} \hat{\mathbf{x}}_{m}(t) \\
& =\boldsymbol{\Phi}_{u}\left(\boldsymbol{\Phi}_{m}^{T} \mathbf{W}^{-1} \boldsymbol{\Phi}_{m}\right)^{-1} \boldsymbol{\Phi}_{m}^{T} \mathbf{W}^{-1} \mathbf{A} \mathbf{x}_{m}(t)
\end{aligned}
$$

If $\boldsymbol{\Phi}_{u}$ is replaced with $\boldsymbol{\Phi}$ in (19) or (22), also the measured DOFs are estimated:

$$
\hat{\mathbf{x}}(t)=\boldsymbol{\Phi} \hat{\mathbf{q}}(t)
$$

which is also used in this study.

\section{NUMERICAL SIMULATIONS}

An experiment was performed with a numerical model of a steel frame (Figure 1) with a height of $4.0 \mathrm{~m}$ and a width of $3.0 \mathrm{~m}$. Both columns were fixed at the bottom. The frame was also supported with a horizontal spring at an elevation of $2.75 \mathrm{~m}$ with a spring constant of 2.0 $\mathrm{MN} / \mathrm{m}$. The frame was modelled with simple beam elements with hollow square cross section of $100 \mathrm{~mm} \times 100 \mathrm{~mm} \times 5 \mathrm{~mm}$. The FE model consisted of 176 beam elements $62.5 \mathrm{~mm}$ in length and a single spring element.

Horizontal random loading was applied to the right column at nodes 113, 129, and 145, corresponding to elevations of $4 \mathrm{~m}, 3 \mathrm{~m}$, and $2 \mathrm{~m}$, respectively (Figure 1). The loads were mutually independent having amplitudes of $9 \mathrm{kN}, 7 \mathrm{kN}$, and $5 \mathrm{kN}$, respectively. All load signals were low-pass filtered below $50 \mathrm{~Hz}$.

Seven first modes were used in the simulation together with a static correction procedure [13]. Modal damping was assumed with a damping ratio of 0.01 for modes $1-2,0.015$ for mode 3 , and 0.02 for modes 4-7.

Transverse displacements were measured at 20 equidistant locations excluding the right hand corner (see Figure 1). Sensor 1 was located at node 9, sensor 2 at node 17, and so on. Sensor 20 was located at node 169. Gaussian noise was added to each sensor. For validation and comparison, exact transverse displacements, rotations, and strains in the whole structure were also computed.

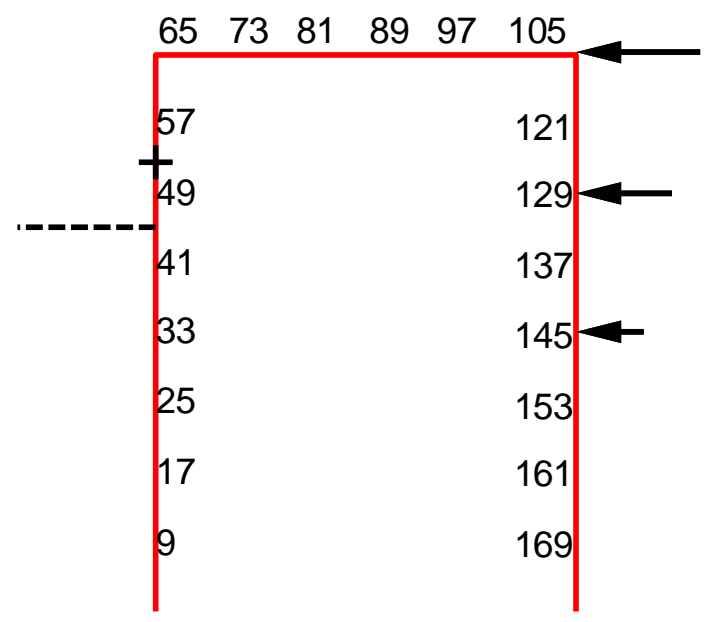

Figure 1: Finite element model and the measurement points (with the corresponding node numbers shown). The + marker shows the location of the virtual strain sensor in the middle of element 52 . 
The same FE model was used for analytical virtual sensing. Ignoring model errors is actually unrealistic, but makes it possible to study the uncertainty reduction due to the measurement error only.

Four different virtual sensing algorithms were studied:

1. Analytical VS using OLS estimation directly from the noisy measurement data.

2. Analytical VS using WLS estimation from the noisy measurement data.

3. Combined VS using OLS estimation from the empirical virtual sensors.

4. Combined VS using WLS estimation from the empirical virtual sensors.

In WLS estimation, the weighting matrix was computed using (13). The following physical displacement sensor sets were used: (1) all 20 sensors; (2) every other sensor, [1:2:20], consisting of ten sensors; (3) the first ten sensors [1:10], and (4) every third sensor, [1:3:20], consisting of seven sensors.

\subsection{All 20 sensors, noise model 1}

First, sensor set 1 including all 20 physical sensors was studied. Four different noise realizations were used: (1) a high signal-to-noise ratio, SNR $=30 \mathrm{~dB}$ in each sensor; (2) a low signal-to-noise ratio, $\mathrm{SNR}=15 \mathrm{~dB}$ in each sensor; (3) equal absolute noise level in each sensor, so that in sensor 1 the signal-to-noise ratio was $\mathrm{SNR}=6 \mathrm{~dB}$; (4) equal absolute noise level in each sensor, so that in sensor 1 the signal-to-noise ratio was $\mathrm{SNR}=-6 \mathrm{~dB}$. Notice that the loading was different in each case, but the results are nevertheless statistically comparable.

In the first noise model, a high signal-to-noise ratio, $\mathrm{SNR}=30 \mathrm{~dB}$ in each sensor was applied. A detail of strain estimates and exact values in the middle of element 52 (left column, elevations $3.2 \mathrm{~m}$ from the ground, see the + marker in Figure 1) are plotted in Figure 2. It can be seen that combined VS was able to reduce noise. Also, WLS resulted in better estimates than OLS.

Uncertainty reduction can also be seen in Figure 3, where the standard deviation of the noise in each sensor is plotted for both the noisy measurements and the empirical virtual sensors. It can be seen that noise could be reduced from all sensors using MMSE estimation, but could not be completely removed.

Figures 4-6 show the relative error of each virtual displacement, rotation, and strain sensor, respectively, on a logarithmic scale. Notice that the physical displacement sensors were also estimated (Equation 23). Because the accuracy of all physical sensors increased due to empirical virtual sensing, the combined VS outperformed analytical VS. Analytical VS using OLS estimation resulted in the most inaccurate estimates. Combined VS using OLS or WLS produced quite similar results, except at the ends of the structure where WLS gave better estimates. Close to the left support, the accuracy of combined VS using OLS was lower than that using analytical VS with WLS. 

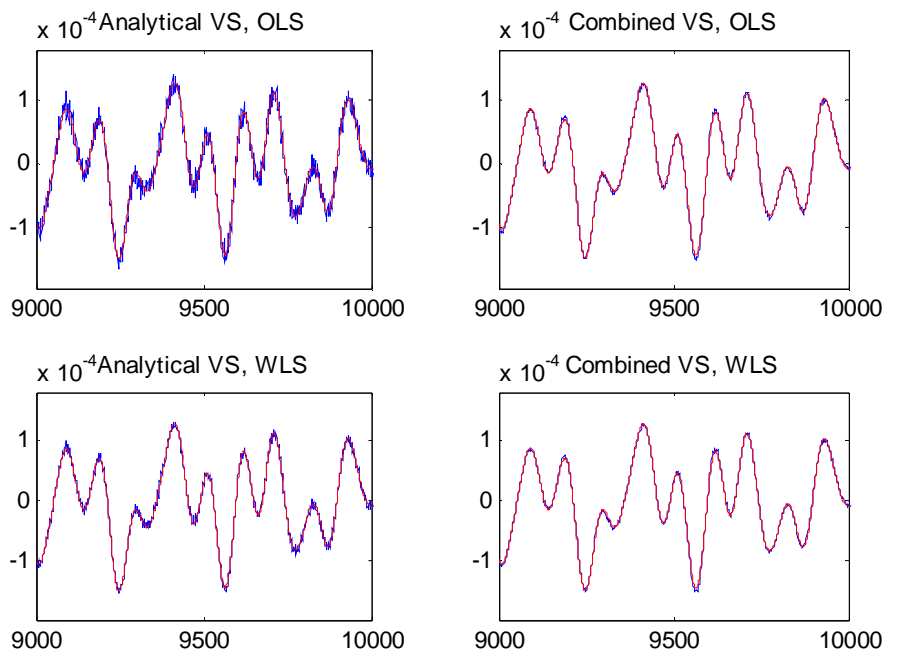

Figure 2: Virtual strain in the middle of element 52 using different algorithms. The red curve is the true strain.

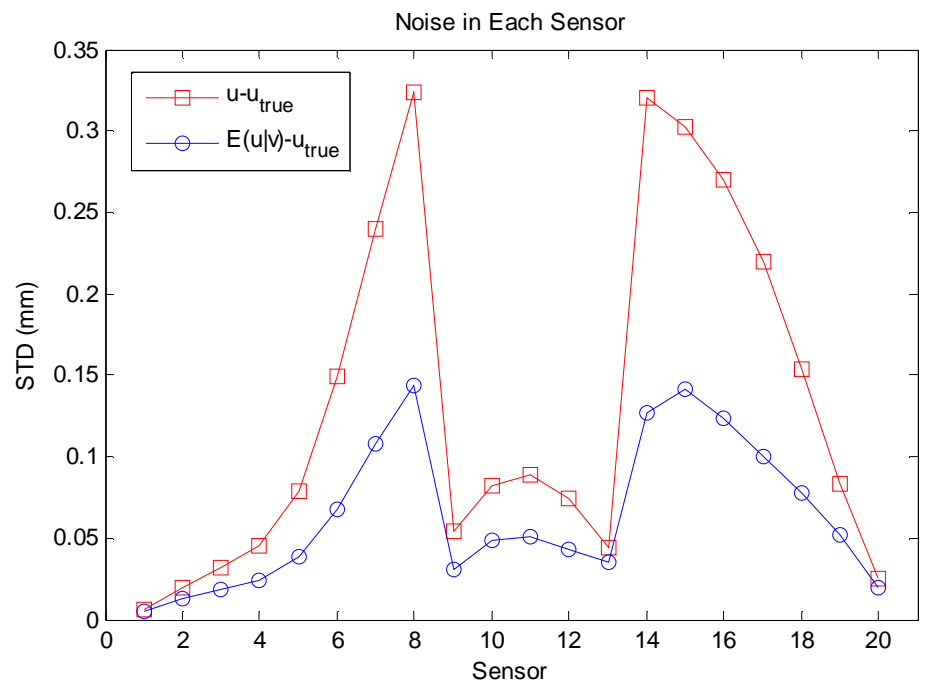

Figure 3: Standard deviations of the noise in each sensor. Measurement data (red) and estimated data (blue).

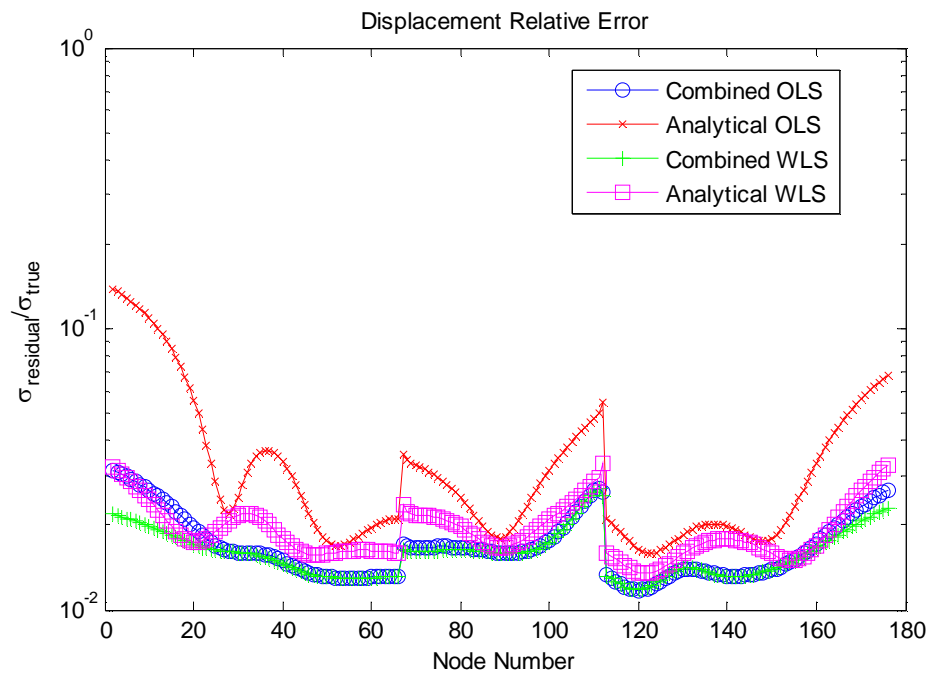

Figure 4: Displacement estimation error of each sensor using four different algorithms. 


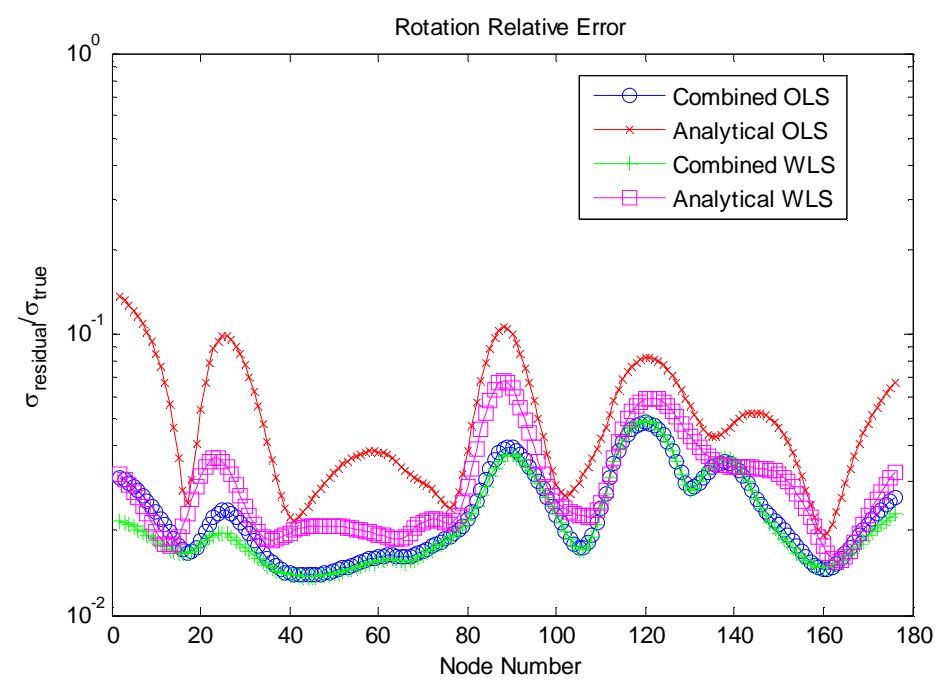

Figure 5: Rotation estimation error of each sensor using four different algorithms.

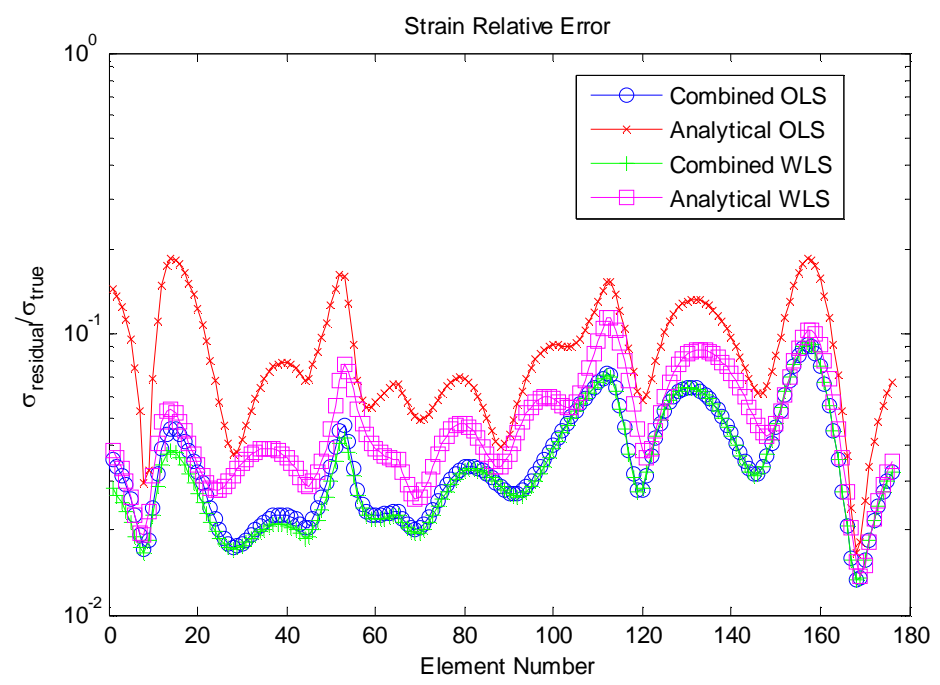

Figure 6: Strain estimation error of each sensor using four different algorithms.

\subsection{All 20 sensors, noise model 2}

The second noise model was the same as in the previous case, but the noise level was higher. Each sensor had a low signal-to-noise ratio $\mathrm{SNR}=15 \mathrm{~dB}$.

Figure 7 shows a detail of virtual strain sensor signal in the middle of element 52 together with the exact red signal. The enhancement of combined VS is clearly visible. Also, WLS produced more accurate strains than OLS.

The standard deviations of the noise in each physical sensor before and after empirical VS are plotted in Figure 8. Empirical VS resulted in more accurate results in all sensors compared to the actual noisy measurements. This gives an indication that combined VS could result in better estimates also for unmeasured DOF.

The relative errors of all virtual displacement, rotation, and strain sensors are shown in Figures 9-11, respectively. The errors are larger than those in the previous case. The improvement of combined VS compared to analytical VS is larger than in the previous lownoise case. Combined VS using WLS outperformed analytical VS. The lowest accuracy was 
obtained using analytical VS with OLS. Again, near the left support, the accuracy of combined VS using OLS was somewhat lower than that using analytical VS with WLS.
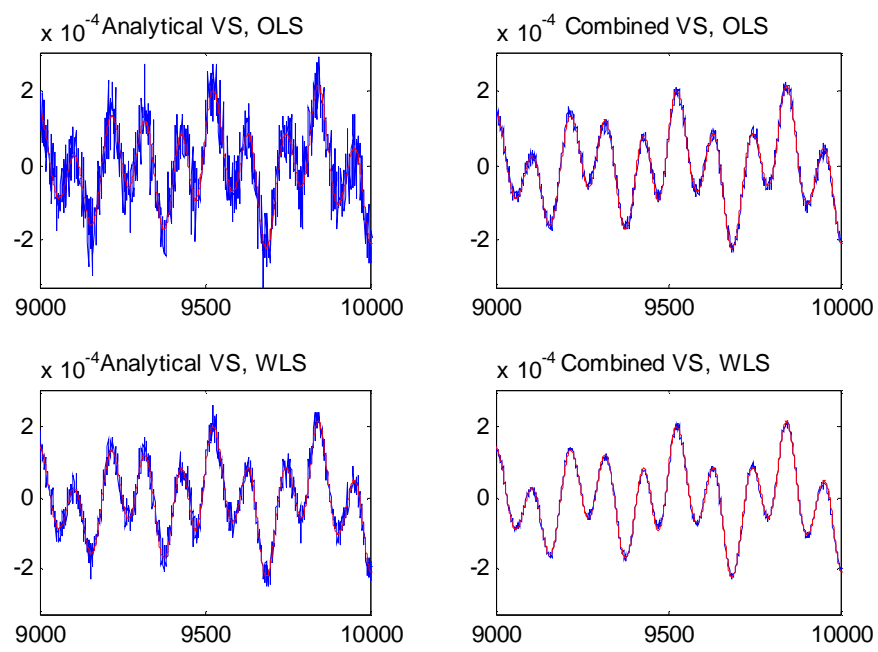

Figure 7: Virtual strain in the middle of element 52 using different algorithms. The red curve is the true strain.

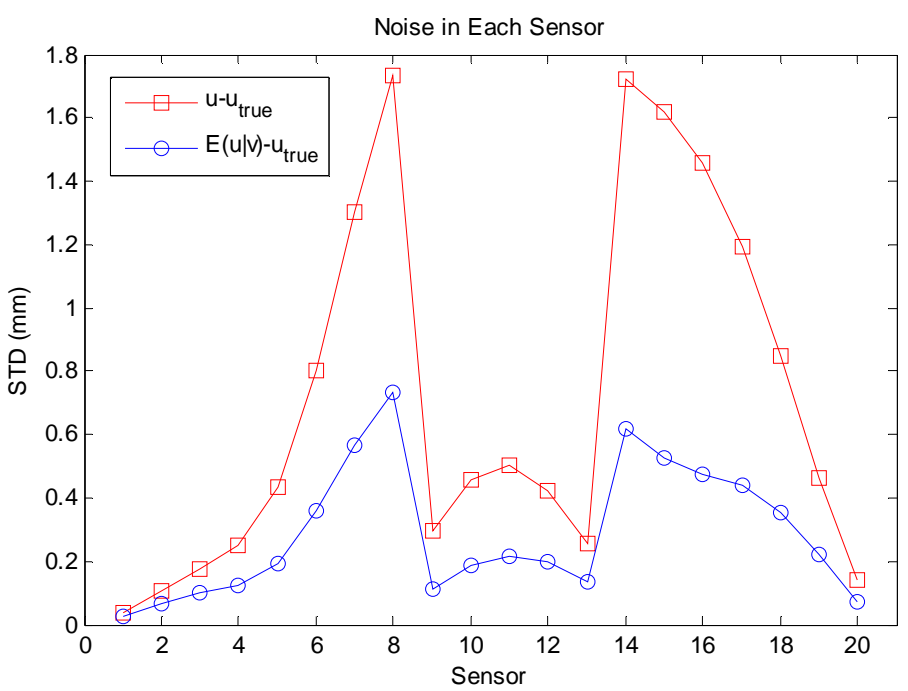

Figure 8: Standard deviations of the noise in each sensor. Measurement data (red) and estimated data (blue). 


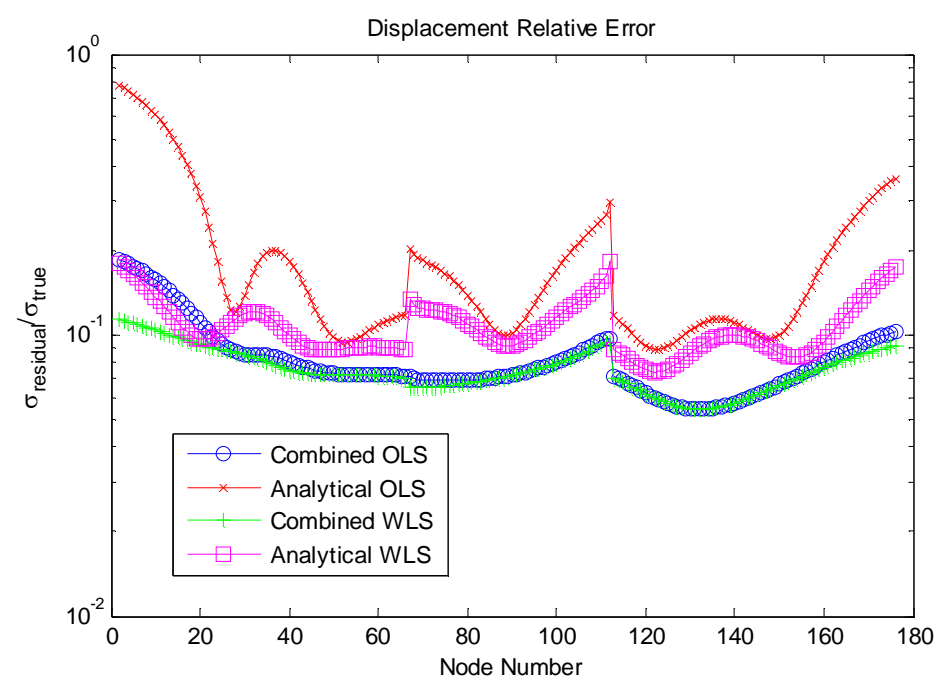

Figure 9: Displacement estimation error of each sensor using four different algorithms.

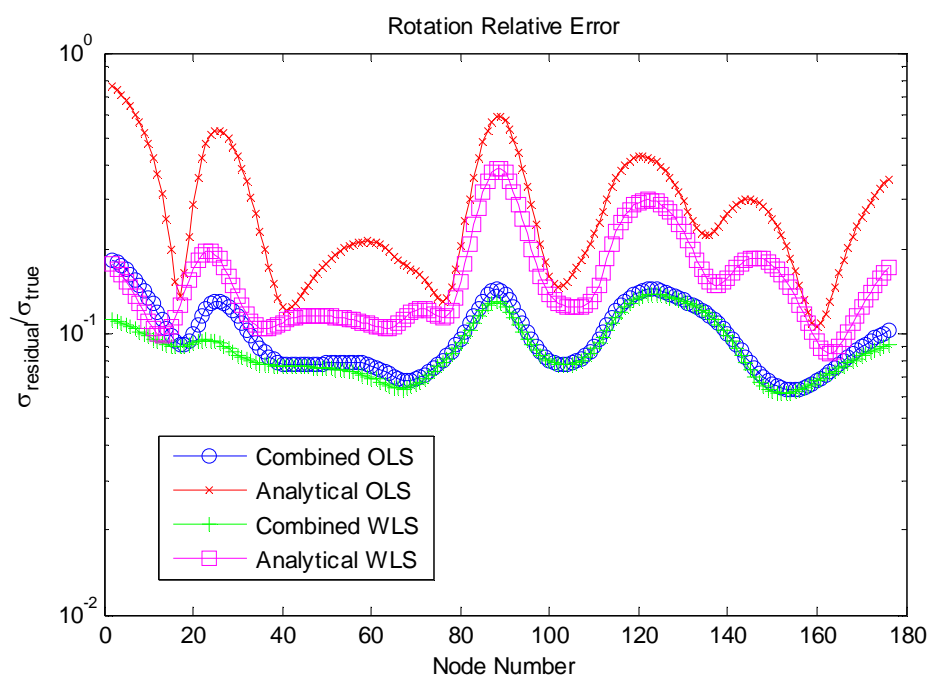

Figure 10: Rotation estimation error of each sensor using four different algorithms.

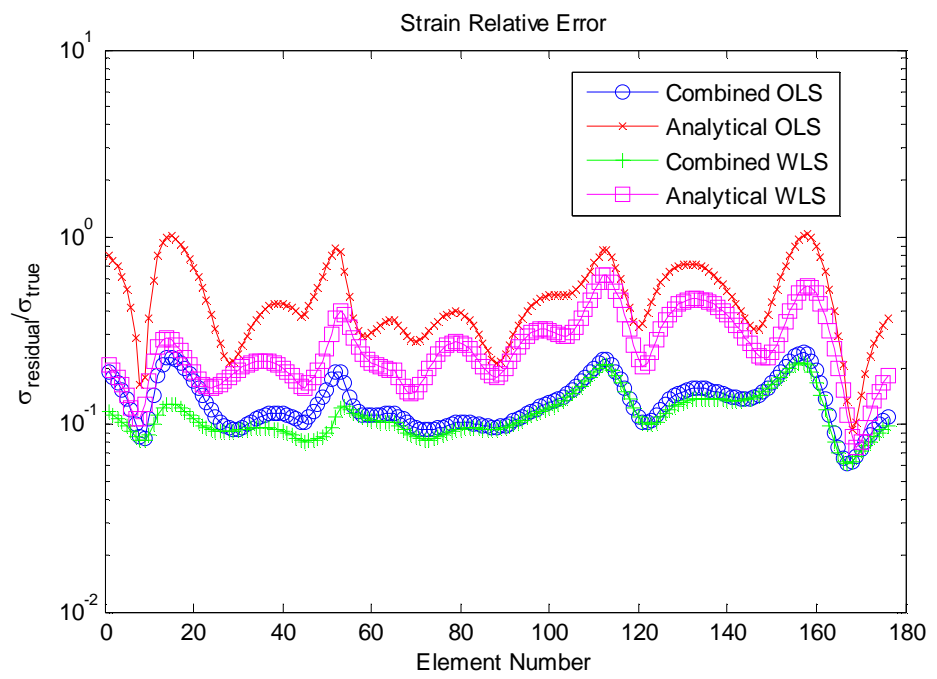

Figure 11: Strain estimation error of each sensor using four different algorithms. 


\subsection{All 20 sensors, noise model 3}

In the third noise model, an equal absolute noise level was introduced to each sensor, determined by assigning a signal-to-noise ratio of $\mathrm{SNR}=6 \mathrm{~dB}$ to sensor 1 .

A sample virtual strain in element 52 using different algorithms is plotted in Figure 12 together with the exact values. Combined VS resulted in more accurate strain measurements. There seem to be no difference between OLS and WLS estimates. This is due to the fact that because the noise variance was equal in all sensors, it resulted in a weighting matrix proportional to the unit matrix $\mathbf{I}$.

Figure 13 shows the standard deviation of the error in each physical sensor and empirical virtual sensor. The constant noise level in each physical sensor is clearly visible. It can be seen that all empirical virtual sensing increased the accuracy of the physical sensors. However, the noise level was now different in these virtual sensors.

The comparison of different algorithms is shown in Figures 14-16, where the relative errors in all virtual displacement, rotation, and strain sensors, respectively, are plotted. OLS and WLS resulted in similar results due to the noise model, as discussed above. Combined VS resulted in more accurate results than analytical VS, with an exception that displacements around node 130 and rotations around nodes 120 and 140 were less accurate. The reason for this is not known, but it is probably related to the low noise level in the physical sensors. In the high-noise case, this phenomenon was not observed, as shown next.
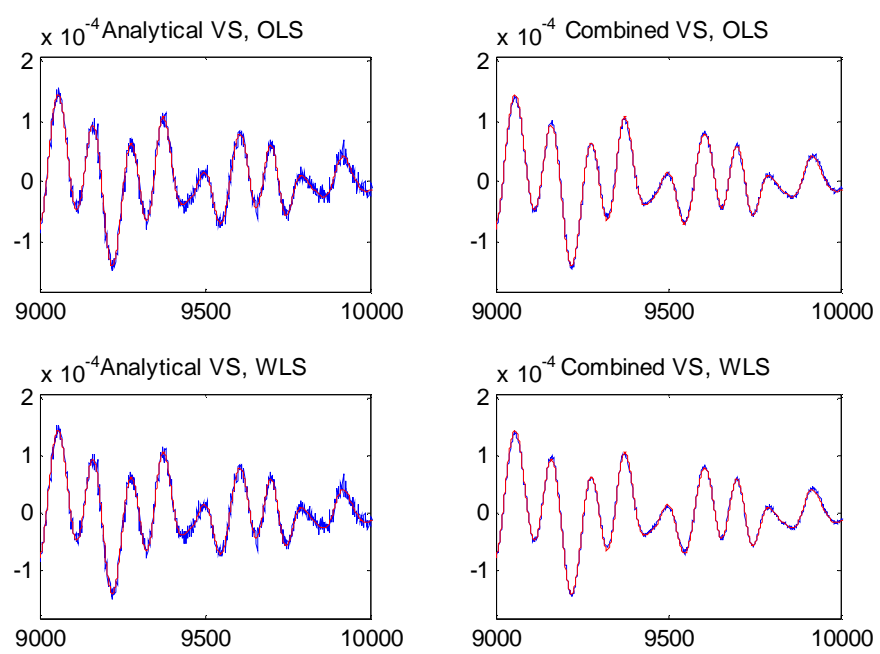

Figure 12: Virtual strain in the middle of element 52 using different algorithms. The red curve is the true strain. 


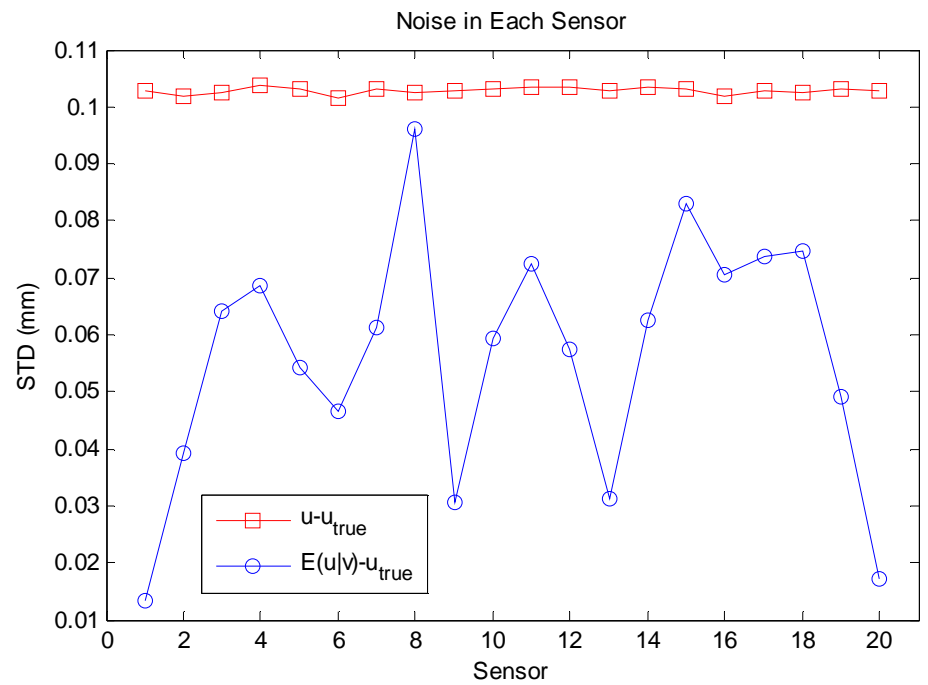

Figure 13: Standard deviations of the noise in each sensor. Measurement data (red) and estimated data (blue).

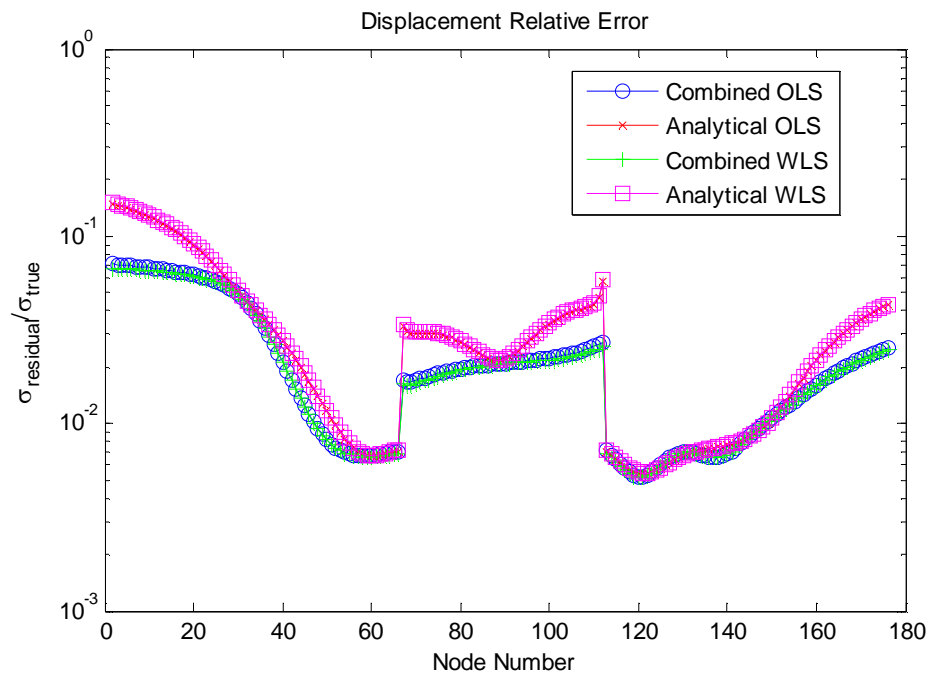

Figure 14: Displacement estimation error of each sensor using four different algorithms.

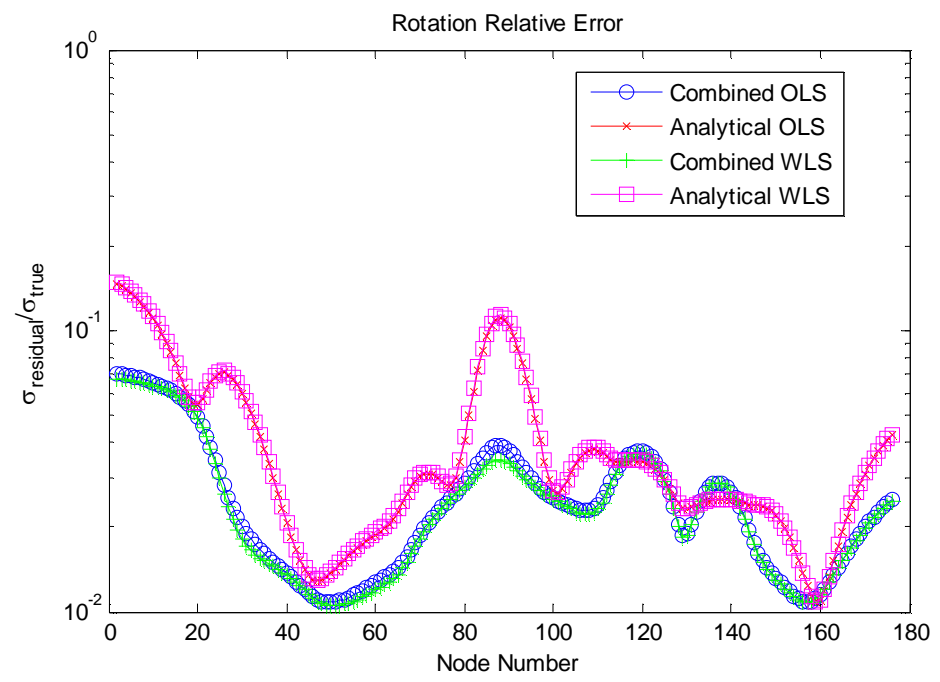

Figure 15: Rotation estimation error of each sensor using four different algorithms. 


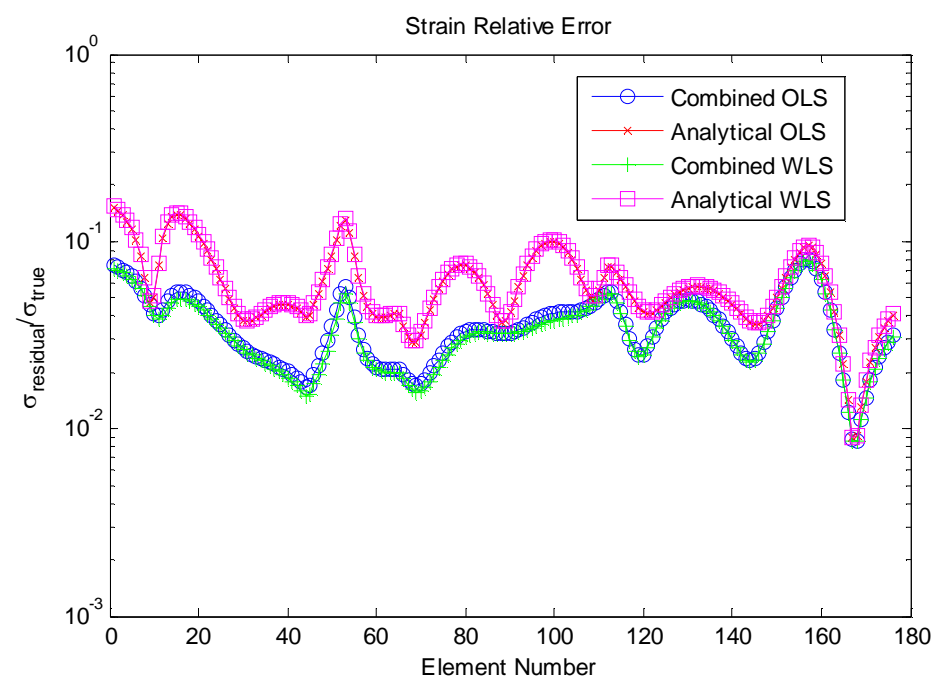

Figure 16: Strain estimation error of each sensor using four different algorithms.

\subsection{All 20 sensors, noise model 4}

The fourth noise model was similar to the previous one, except that now the noise level was higher. Each sensor had equal absolute noise level, determined by assigning a signal-tonoise ratio of $\mathrm{SNR}=-6 \mathrm{~dB}$ to sensor 1 .

Figure 17 shows a detail of virtual strain in the middle of element 52. Again, the noise reduction of the combined VS is clearly visible. OLS and WLS gave similar results due to the fact that the weighting matrix was proportional to the unit matrix $\mathbf{I}$.

Empirical VS was able to reduce noise in all sensors, which is shown in Figure 18, where the noise level of each physical and corresponding virtual sensor is plotted. After empirical VS, the noise level in each sensor was different.

Figures 19-21 show the relative errors of virtual displacement, rotation, and strain sensors, respectively. Combined VS outperformed analytical VS in each virtual sensor. OLS and WLS produced similar results due to the applied noise model, as discussed above.

As a conclusion, combined VS seems to be able to reduce noise and result in more accurate estimates than analytical VS. The difference was more dominant if the noise level of the physical sensors was high. WLS outperformed OLS in cases where the noise level in each physical sensor was different. If the noise level was equal in each sensor, either method could be used. Thus WLS should be always preferred.

Empirical VS was able to reduce noise of the physical sensors in all cases so far. The reason is assumed to be the high number of sensors, which makes it possible to gather more data to obtain better estimates. Next, different sensor networks are studied using a smaller number of physical sensors at different locations of the structure. 

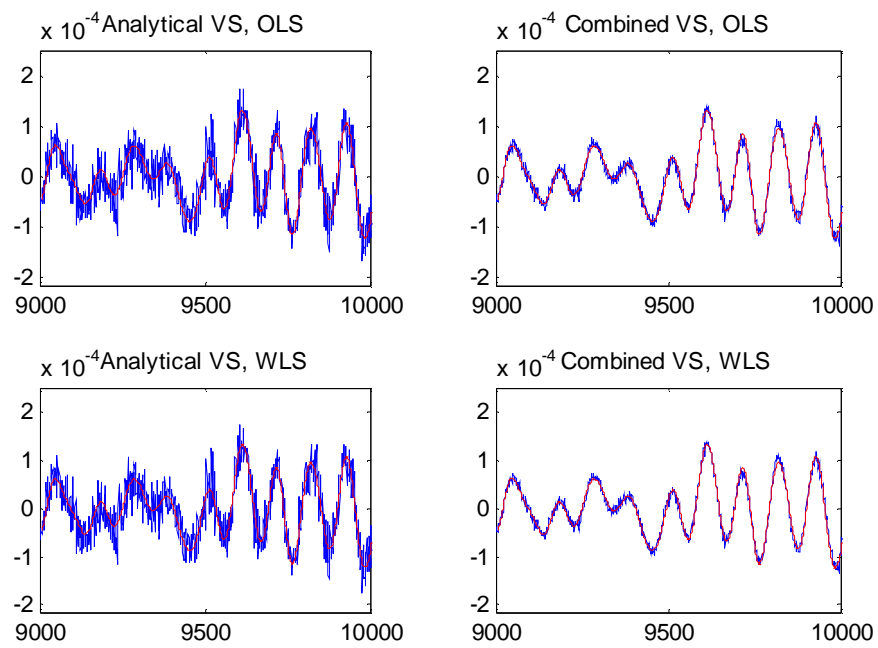

Figure 17: Virtual strain in the middle of element 52 using different algorithms. The red curve is the true strain.

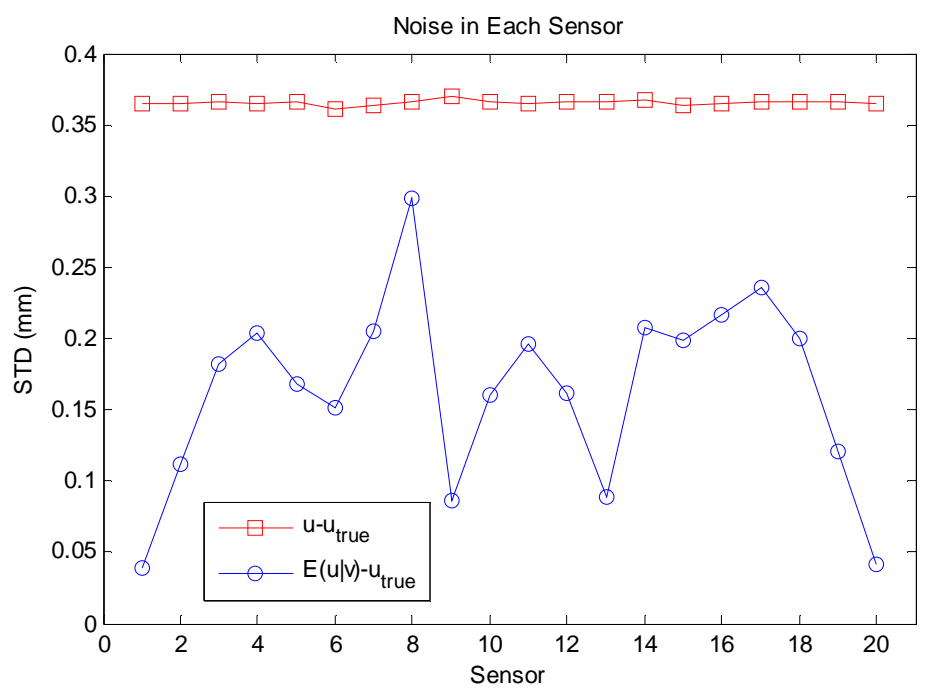

Figure 18: Standard deviations of the noise in each sensor. Measurement data (red) and estimated data (blue).

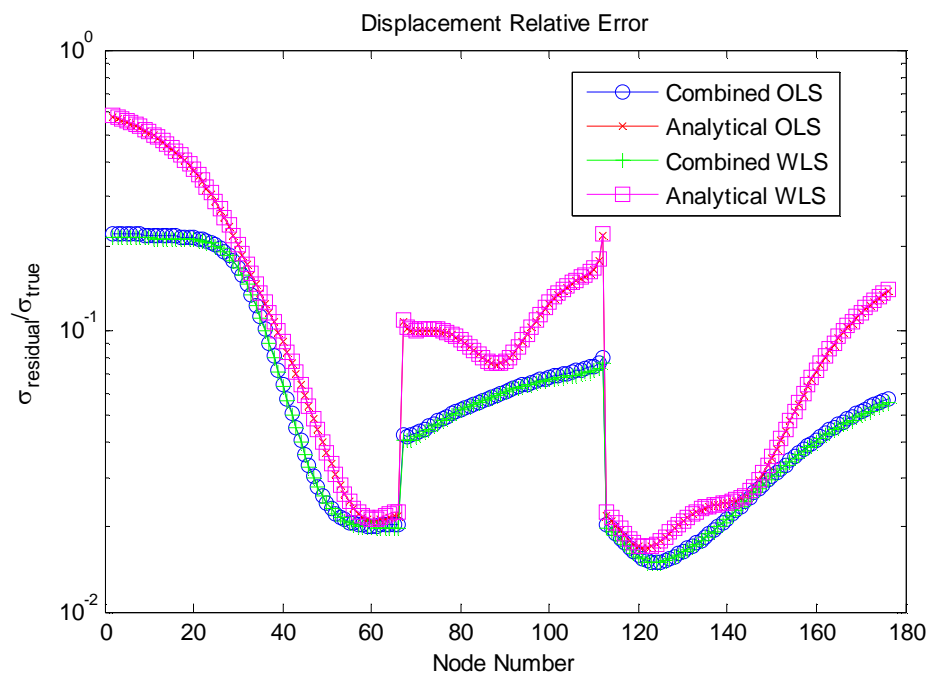

Figure 19: Displacement estimation error of each sensor using four different algorithms. 


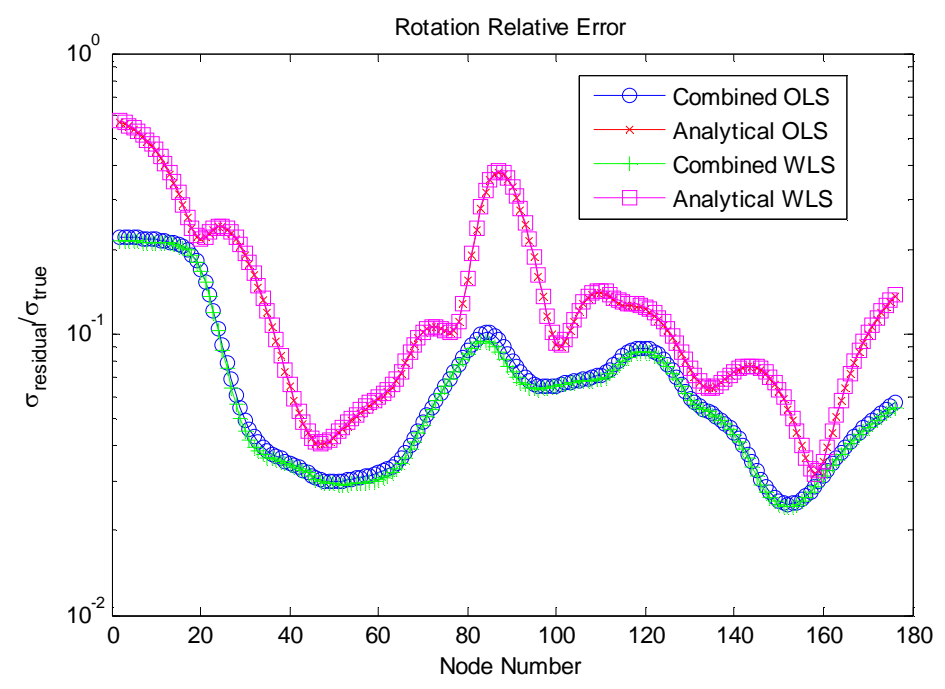

Figure 20: Rotation estimation error of each sensor using four different algorithms.

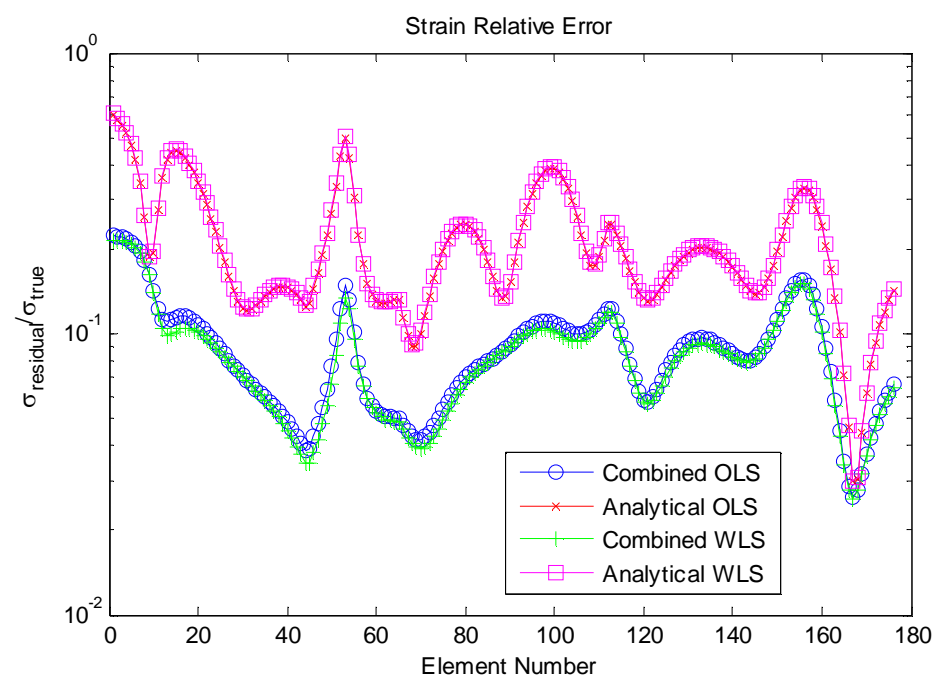

Figure 21: Strain estimation error of each sensor using four different algorithms.

\subsection{Sensor set 2}

Sensor sets 2-4 were studied using noise model 1 . Sensor set 2 included every other sensor [1:2:20], ten sensors in total. Figure 22 shows sample virtual strain estimates in the middle of element 52 using all four algorithms together with the exact strain. Combined VS was able to reduce noise, but the performance is not very pronounced.

Figure 23 shows the noise level in each physical sensor and the corresponding empirical virtual sensor. Noise reduction due to empirical VS was significant in three sensors, but sensor 19 and sometimes sensors 1 or 13 were less accurate after estimation. In reality, the exact measurement is not available, and thus it is not known which sensors benefit analytical VS.

Relative error of all virtual displacement, rotation, and strain sensors are shown in Figures 24-26, respectively, using different algorithms. Combined VS resulted in the most accurate signals in most virtual sensors. However, in some sensors, combined VS with OLS or analytical VS with WLS resulted in higher accuracy. Analytical VS with OLS resulted in the most inaccurate estimations. 
As a comparison, in a high-noise case (not shown), combined VS using WLS outperformed the other algorithms.
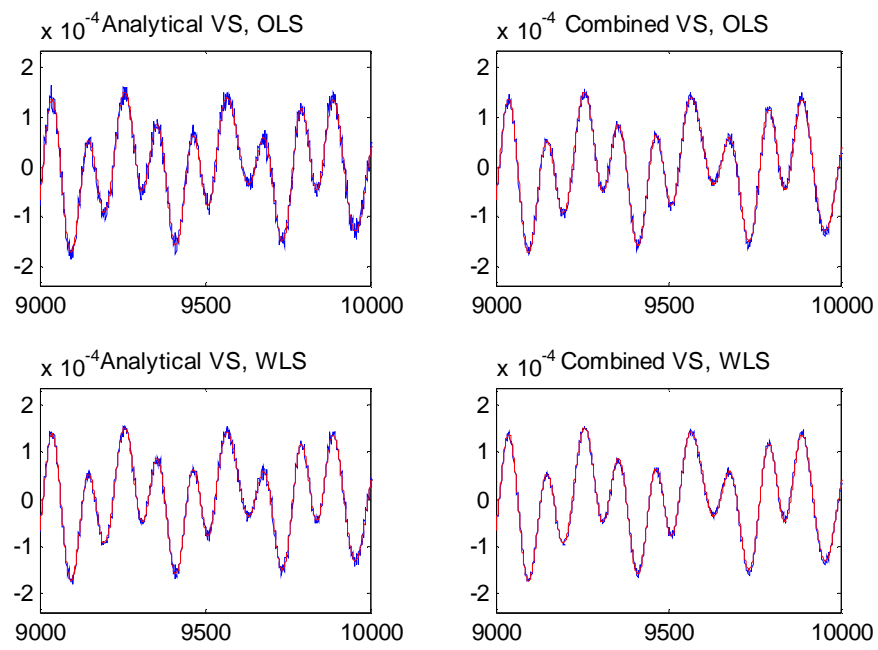

Figure 22: Virtual strain in the middle of element 52 using different algorithms. The red curve is the true strain.

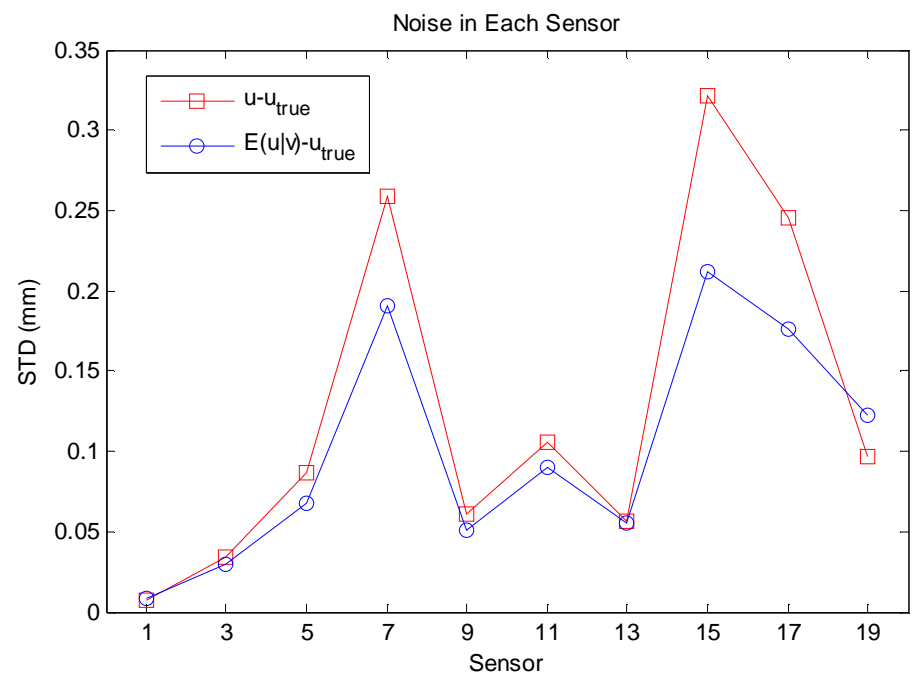

Figure 23: Standard deviations of the noise in each sensor. Measurement data (red) and estimated data (blue). 


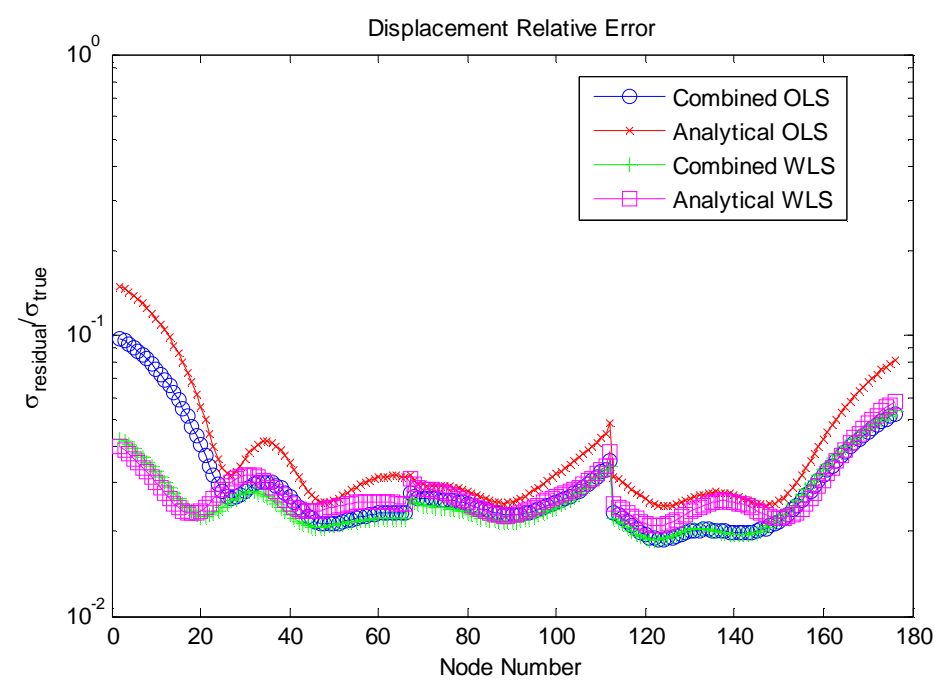

Figure 24: Displacement estimation error of each sensor using four different algorithms.

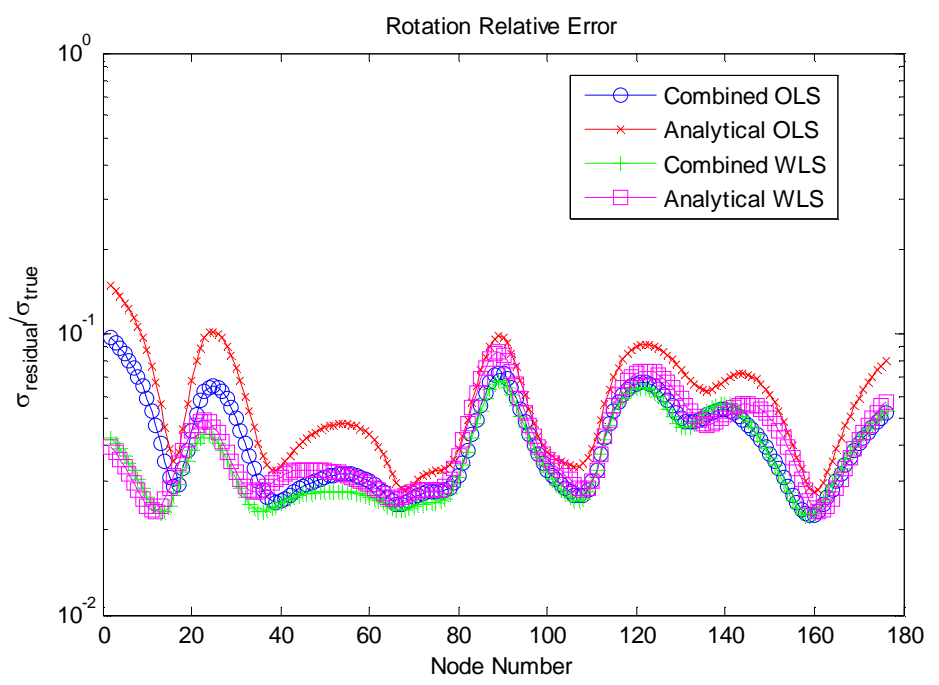

Figure 25: Rotation estimation error of each sensor using four different algorithms.

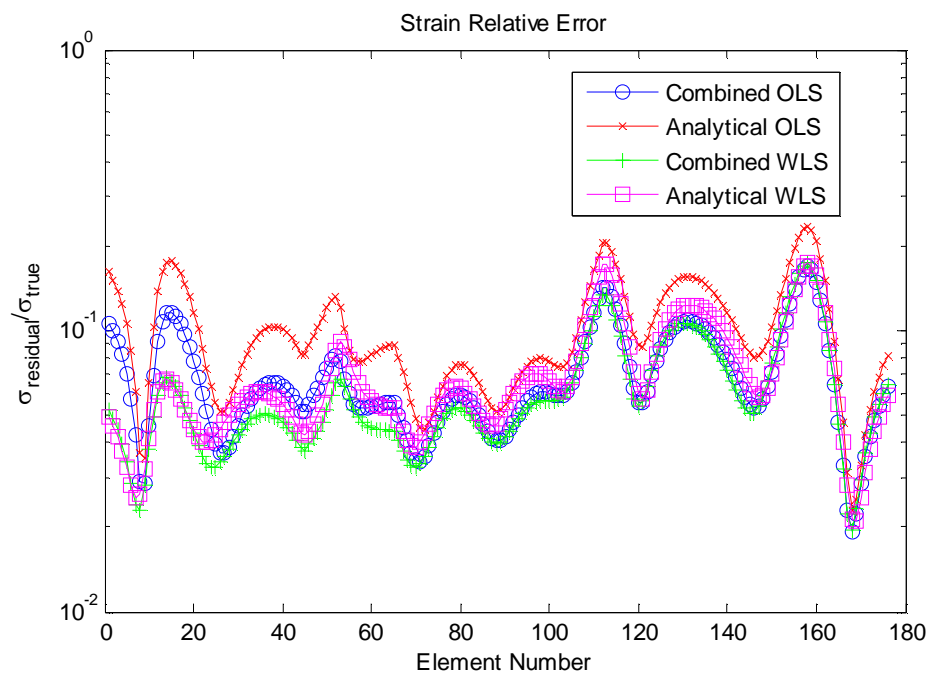

Figure 26: Strain estimation error of each sensor using four different algorithms. 


\subsection{Sensor set 3}

Also sensor set 3 included ten physical sensors, which were the first ten sensors. The right hand side of the structure was uninstrumented.

Figure 27 shows a significant noise reduction performance of combined VS with WLS estimation in the virtual strain sensor in the middle of element 52 compared to analytical VS using OLS estimation.

The error in each physical sensor and the corresponding empirical virtual sensor is plotted in Figure 28. Empirical virtual sensing improved accuracy of all physical sensors except sensor 10 .

Relative errors in all virtual displacement, rotation, and strain sensors are shown in Figures 29-31. Combined VS using WLS outperformed the other algorithms, especially analytical VS using OLS, which produced the most inaccurate estimates, with almost a decade lower relative error. The accuracy of virtual sensors in the uninstrumented part of the structure was very low. This suggests that physical sensors should be installed uniformly all around the structure, especially close to the virtual sensors.
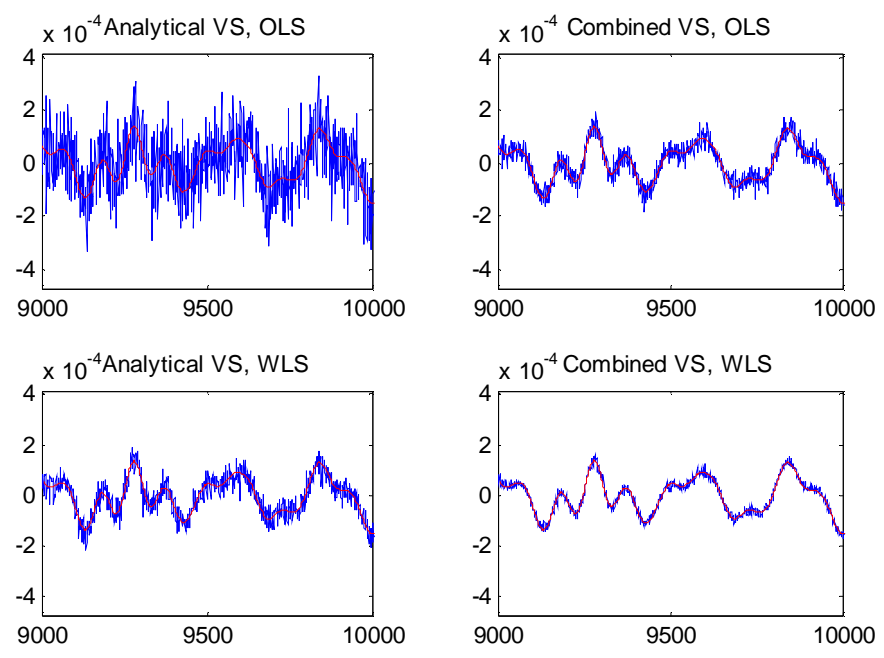

Figure 27: Virtual strain in the middle of element 52 using different algorithms. The red curve is the true strain.

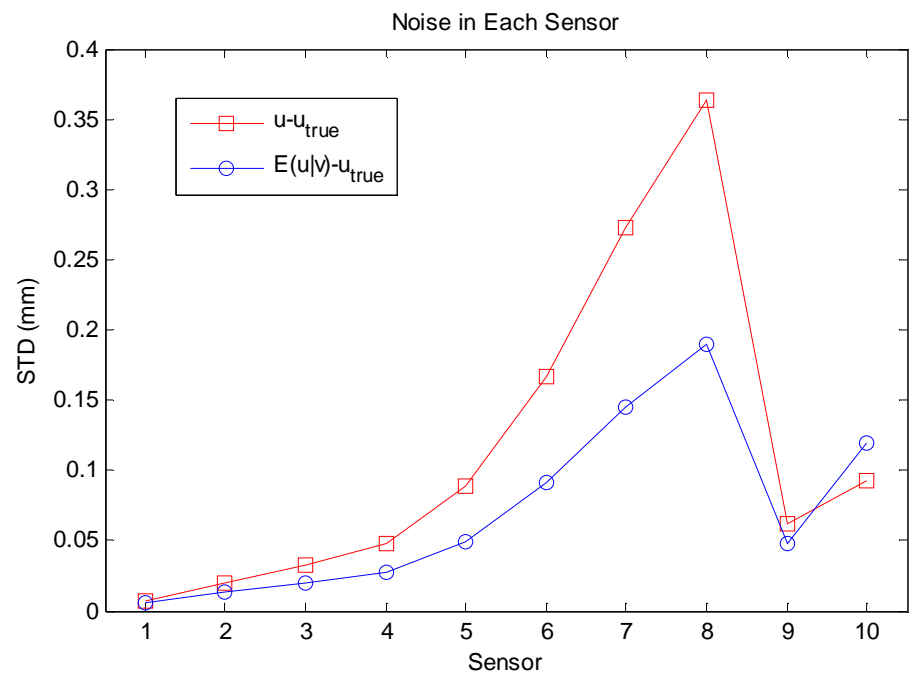

Figure 28: Standard deviations of the noise in each sensor. Measurement data (red) and estimated data (blue). 


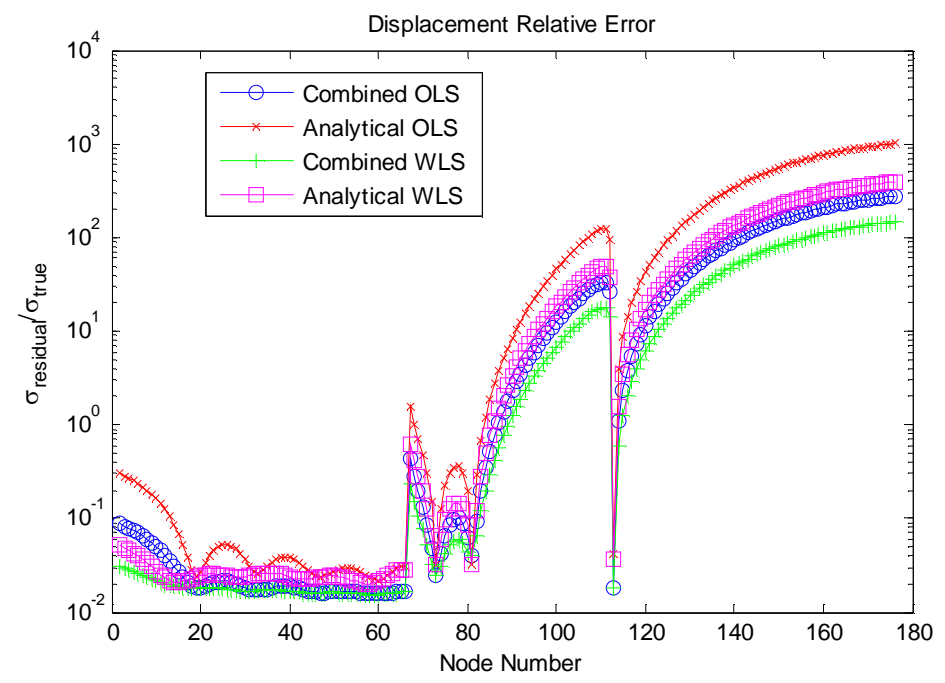

Figure 29: Displacement estimation error of each sensor using four different algorithms.

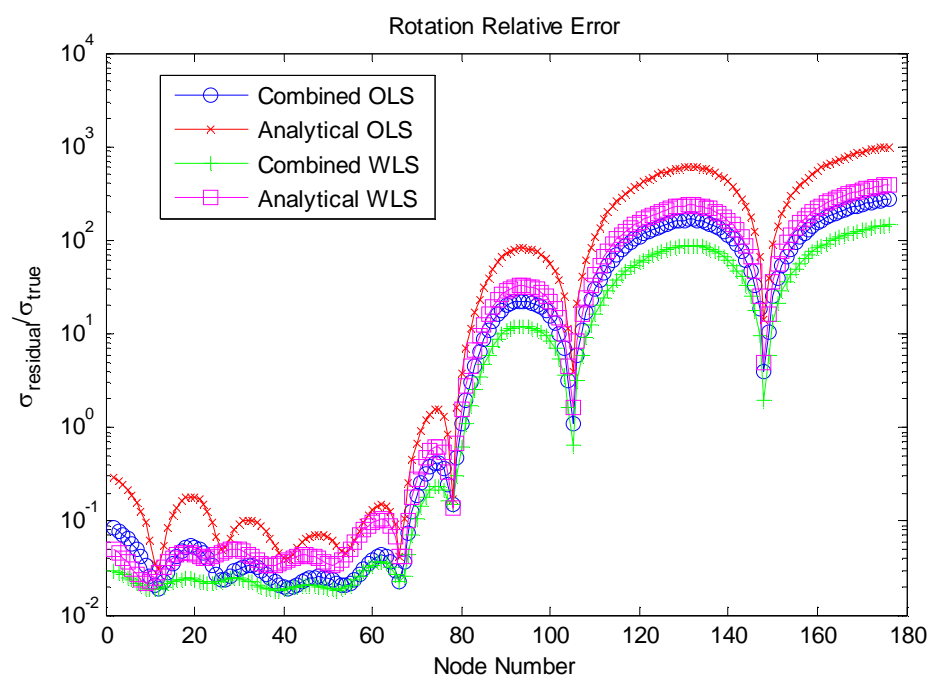

Figure 30: Rotation estimation error of each sensor using four different algorithms.

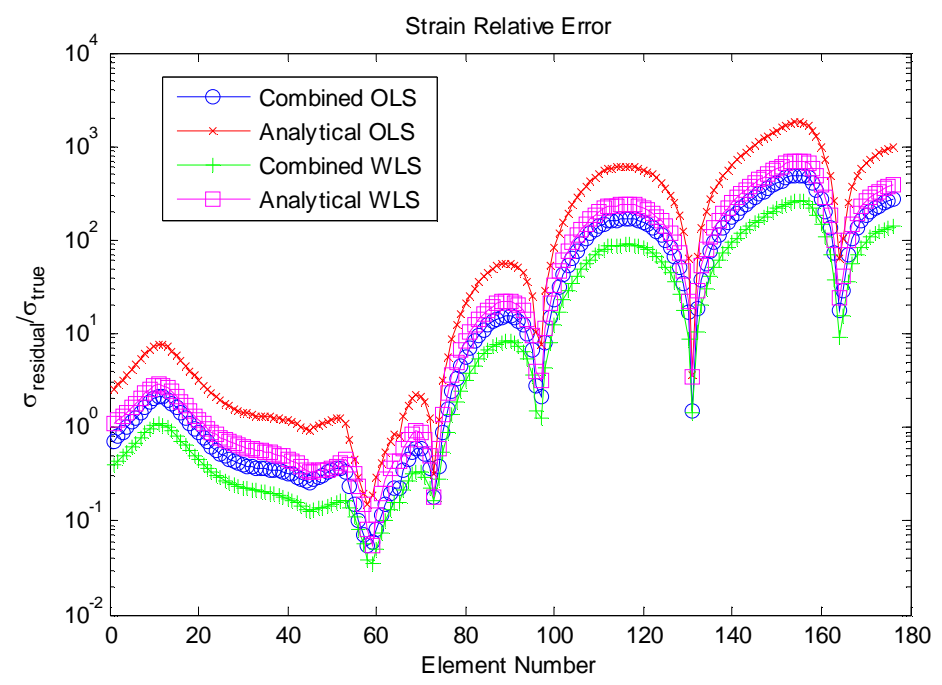

Figure 31: Strain estimation error of each sensor using four different algorithms. 


\section{7 $\quad$ Sensor set 4}

Sensor set 4 included every third sensor [1:3:20], including only seven physical sensors.

Figure 32 shows virtual strain in the middle of element 52 together with the exact strain. Comparison of different algorithms suggests that there are no distinct differences in accuracy.

The relative error in each physical sensor and in the corresponding virtual sensor are shown in Figure 33. Empirical VS was able to improve the accuracy in two sensors only. In the remaining five sensors, the noise increased, but fortunately the precision degradation was quite small.

The relative error in each virtual displacement, rotation, and strain sensor is plotted in Figures 34-36, respectively. The ranking of the algorithms is difficult, because at some nodes, analytical VS performed better than combined VS and at the remaining nodes combined VS resulted in better estimations. However, the differences were quite small. OLS and WLS produced similar results. This is probably due to the fact that the redundancy was quite weak.

As a comparison, in a high-noise case (not shown), combined VS performed better than analytical VS, because empirical VS resulted in better noise reduction.
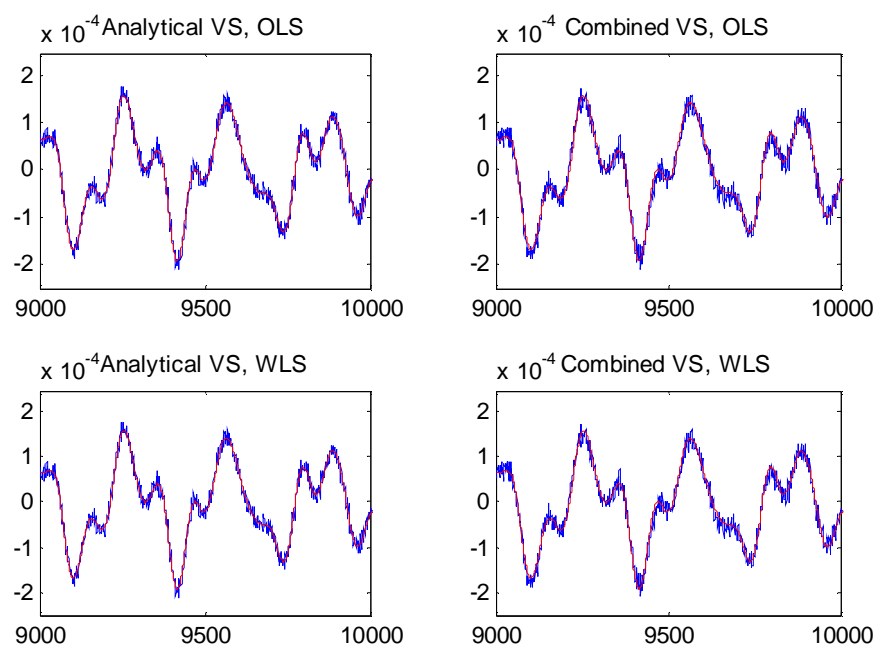

Figure 32: Virtual strain in the middle of element 52 using different algorithms. The red curve is the true strain.

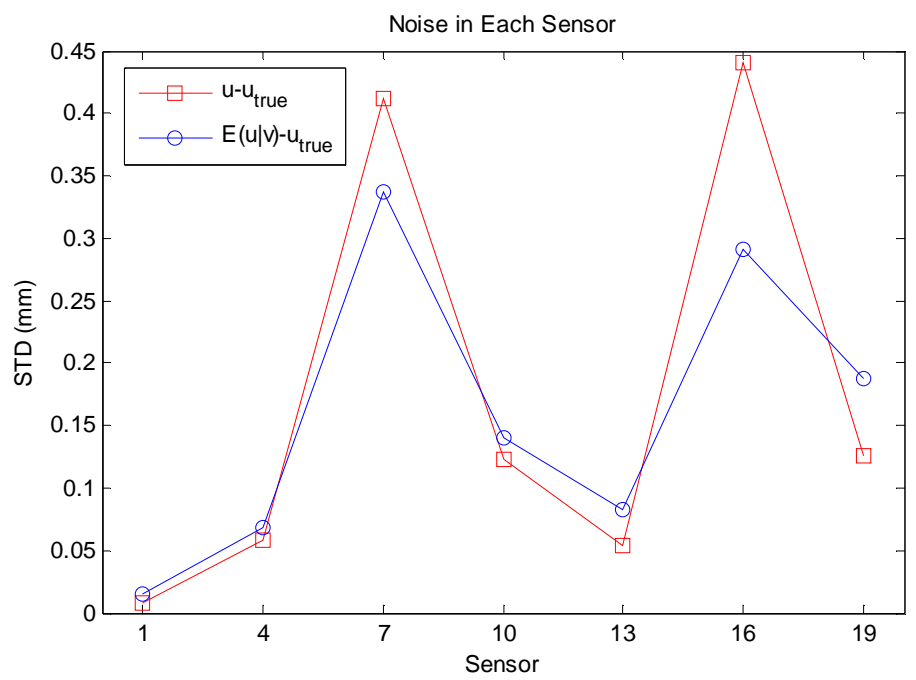

Figure 33: Standard deviations of the noise in each sensor. Measurement data (red) and estimated data (blue). 


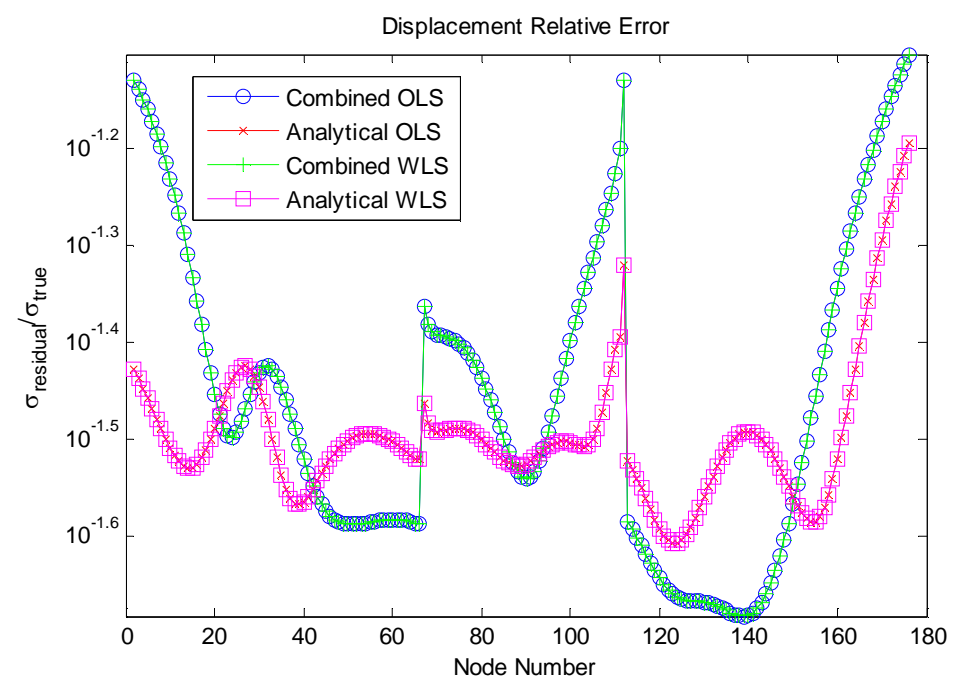

Figure 34: Displacement estimation error of each sensor using four different algorithms.

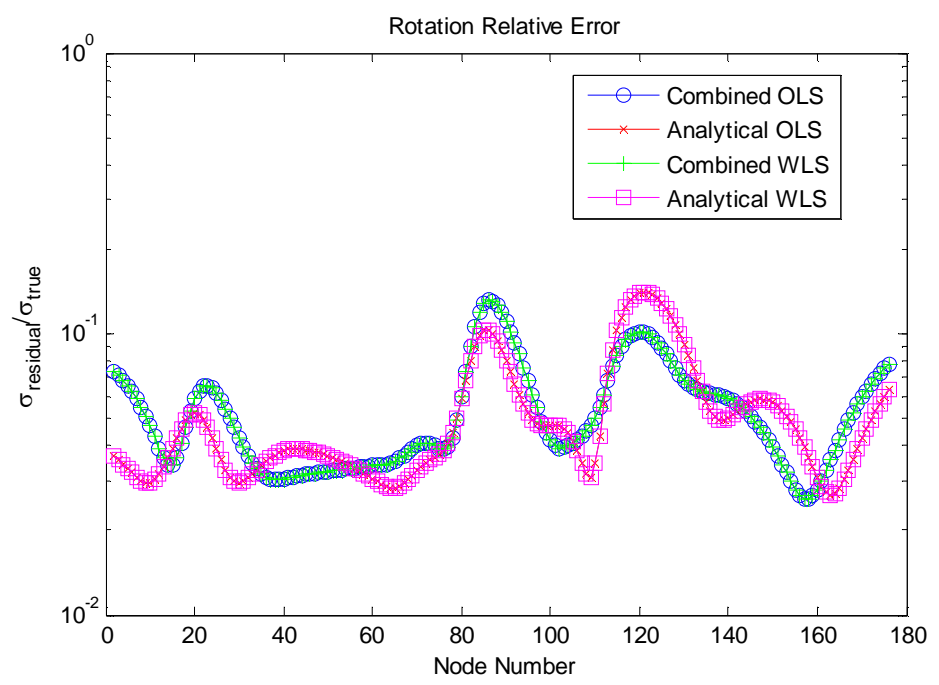

Figure 35: Rotation estimation error of each sensor using four different algorithms.

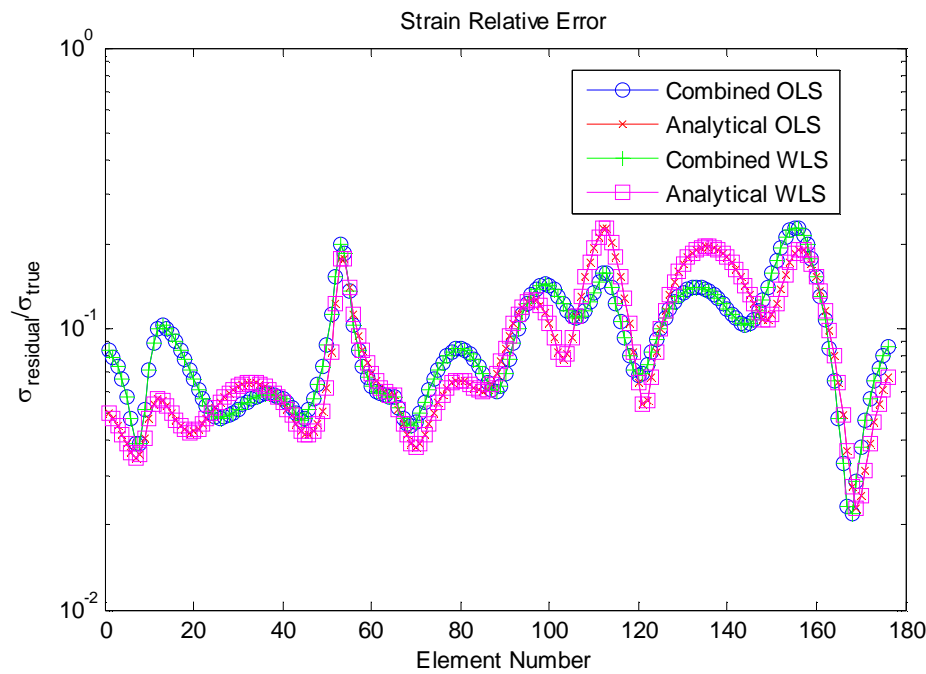

Figure 36: Strain estimation error of each sensor using four different algorithms. 


\section{CONCLUSIONS}

A combined empirical and analytical virtual sensing algorithm is proposed for structural dynamics. Uncertainty reduction can be obtained by (1) Replacing noisy measurements with less noisy MMSE estimates before analytical virtual sensing, and (2) applying WLS in analytical virtual sensing with the weighting matrix W obtained from the MMSE algorithm.

In the case with a large sensor network with 20 sensors, the accuracy of the MMSE estimates was always higher than that of the actual measurements regardless of the noise level or noise model. This property assured uncertainty reduction also in the virtual sensors. With a smaller sensor network (10 sensors), the redundancy was weaker, resulting in cases in which a small number (1-2) of empirical virtual sensors exhibited higher error than the actual measurements. However, combined VS using WLS was always more accurate than the analytical VS using OLS. With seven sensors only, analytical VS resulted sometimes in more accurate estimations. In the uninstrumented part of the structure, the estimation errors were very large using any algorithm. Physical sensors should therefore be installed close to the virtual sensors.

In practice, the exact signal is not available. Therefore, it is generally not known, if empirical VS results in a more accurate sensor reading for all sensors. This study suggested that two conditions should be fulfilled when using empirical VS: (1) Redundancy: If the number of sensors in the network is large compared to the number of active modes, it is possible to have an accurate estimation of all sensors. (2) Noisy data: If the noise level is high, empirical VS is recommended. With a low noise level, a small number of virtual sensors was estimated more accurately using analytical VS. A more precise statement of conditions remains an open question.

In this study, model error was not present, which is an unrealistic assumption. The model errors were ignored in order to investigate the noise effect only. The effect of model errors is left for further study.

Environmental or operational variability causes no problem in empirical virtual sensing. However, in analytical virtual sensing, the mode shapes may change due to the aforementioned variability and thus increase the model error. Although the model error was not studied in this paper, it should be noticed that it also affects the estimation accuracy of the unmeasured quantities.

Simultaneous sampling and a redundant sensor network were assumed. Stationarity assumption is not necessary, provided the number of active modes is smaller than the number of sensors in the network. Spatial correlation was only used between sensors at same time instants. This corresponds to snapshots of the structure, which takes no temporal correlation into consideration. For spatiotemporal correlation, the assumption of stationarity may be necessary. Spatiotemporal correlation is left for further study.

\section{REFERENCES}

[1] B. Lin, B. Recke, J.K.H. Knudsen, S.B. Jørgensen, A systematic approach for soft sensor development. Computers and Chemical Engineering, 31, 419-425, 2007.

[2] P. Avitabile, Model Reduction and Model Expansion and Their Applications - Part 1: Theory. Proceedings of IMAC-XXIII, A Conference \& Exposition on Structural Dynamics, Orlando, Florida, USA, January 31-February 3, 2005.

[3] A. Sestieri, W. D'Ambrogio, R. Brincker, A. Skafte, A. Culla, Estimation of Rotational Degrees of Freedom by EMA and FEM Mode Shapes. R. Allemang, J. De Clerck, C. 
Niezrecki, A. Wicks eds. Special Topics in Structural Dynamics, Volume 6, Proceedings of the 31st IMAC, A Conference on Structural Dynamics, 2013.

[4] H.P. Hjelm, R. Brincker, J. Graugaard-Jensen, K. Munch, Determination of stress histories in structures by natural input modal analysis. Proceedings of IMAC-XXIII, A Conference \& Exposition on Structural Dynamics, Orlando, Florida, USA, January 31February 3, 2005.

[5] A. Iliopoulos, C. Devriendt, P. Guillaume, D. Van Hemelrijck, Continuous fatigue assessment of an offshore wind turbine using a limited number of vibration sensors. V. Le Cam, L. Mevel, F. Schoefs eds. EWSHM - 7th European Workshop on Structural Health Monitoring, Nantes, France, July 8-11, 2014.

[6] P. Pingle, P. Avitabile, Full-field dynamic stress/strain from limited sets of measured data. Sound and Vibration, 10-14, August 2011.

[7] E. Harvey, J. Ruddock, P. Avitabile, Comparison of full field strain distributions to predicted strain distributions from limited sets of measured data for SHM applications. B. Basu ed. Tenth International Conference on Damage Assessment to Structures (DAMAS 2013), Dublin, Ireland, July 8-10, 2013.

[8] K. Maes, G. De Roeck, G. Lombaert, Response estimation in structural dynamics. A. Cunha, E. Caetano, P. Ribeiro, G. Müller eds. Proceedings of the 9th International Conference on Structural Dynamics, EURODYN 2014, Porto, Portugal, June 30-July 2, 2014.

[9] L. Liu, S.M. Kuo, M. Zhou, Virtual sensing techniques and their applications. Proceedings of the 2009 IEEE International Conference on Networking, Sensing and Control, Okayama, Japan, March 26-29, 2009.

[10] J. Kullaa, Sensor validation using minimum mean square error estimation. Mechanical Systems and Signal Processing, 24, 1444-1457, 2010.

[11] J. Kullaa, J. 2014. Structural health monitoring under nonlinear environmental or operational influences. Shock and Vibration, 2014, 2014.

[12] Y. Bar-Shalom, X.-R. Li, T. Kirubarajan, Estimation with applications to tracking and navigation. Wiley, 2001.

[13] R.W. Clough, J. Penzien, Dynamics of structures. 2nd edition. McGraw-Hill, 1993. 